\title{
Blockchain: Proposed Architecture and It's Applications
}

\author{
Megha Bansal
}

Department of Information Technology, Assistant Professor, Vivekananda Institute of Professional Studies, India.

To Cite this Article

Megha Bansal, "Blockchain: Proposed Architecture and It's Applications", International Journal for Modern Trends in Science and Technology, 6(8): 196-202, 2020.

\section{Article Info}

Received on 14-July-2020, Revised on 02-August-2020, Accepted on 06-August-2020, Published on 15-August-2020.

\section{ABSTRACT}

Blockchain technology, which is known as the basis of bitcoin and various other crypto currencies, is nowadays having enormous attention. The basis of this technology lies in immutable ledger that allows the transactions to occur in a decentralized manner which provides anonymity to the customers. The current paper gives an overview of the blockchain technology; its process flow and various types or categories. Architecture on blockchain technology has been proposed, which can be used on almost every domain of it. Also a comparison of various architectures that have been proposed previously has been carried out based on some identified parameters. Finally at the end various application areas of the technology have been discussed.

KEYWORDS: Anonymity, Blockchain, Bitcoin, Consensus Protocol

\section{INTRODUCTION}

Blockchain is a technology which is used for the creation of many crypto currencies like bitcoin by the usage of a digital decentralized ledger over a Peer-to-Peer (P2P) network. All the participants are permitted to do the changes with ledger under the usage of blockchain protocols. Blockchain is basically made up of chain of blocks which further results into an open source, immutable, public digital ledger. This technology was coined by Nakamoto (2008) in the context of a peer to peer electronic cash system titled - "Bitcoin". This fundamental study shows the potential of a new budding peer-to-peer technology without the help of any operating central authority or bank instead collectively the network itself manages the transactions and the issuing of bitcoins. Main thought of Nakamoto relied on a multi-signature feature to approve every transactions after which permission from other members has been taken and a full audit trail identifying which members permitted each particular transaction [1].

Major attraction of blockchain is that it does not require any intermediary central authority or third party for transactions over peer-to-peer network. Many cryptocurrencies were prepared out of the concept of blockchain since this technology provides features like immutability, transparency, anonymity, security and decentralization which also lead to expanding it towards more and more fields of the industry.

The blockchain process begins with a transaction request generated by any user. Next this request is broadcasted to the network. A new block of data is created by obtaining a certain number of approved nodes; this is called reaching consensus. After consensus attainment, new "block" on the entire blockchain network is formed, and every node updates its respective copy of the blockchain ledger. New block is linked to the original block 
through digital signature, which completes the transaction process. Further all participants add the new block to their respective ledgers only after validation process, after this each participant contains a replica of new block with it so that every change to the ledger is reflected across the entire network and each network member has a full and same copy of the entire ledger at any point in time.

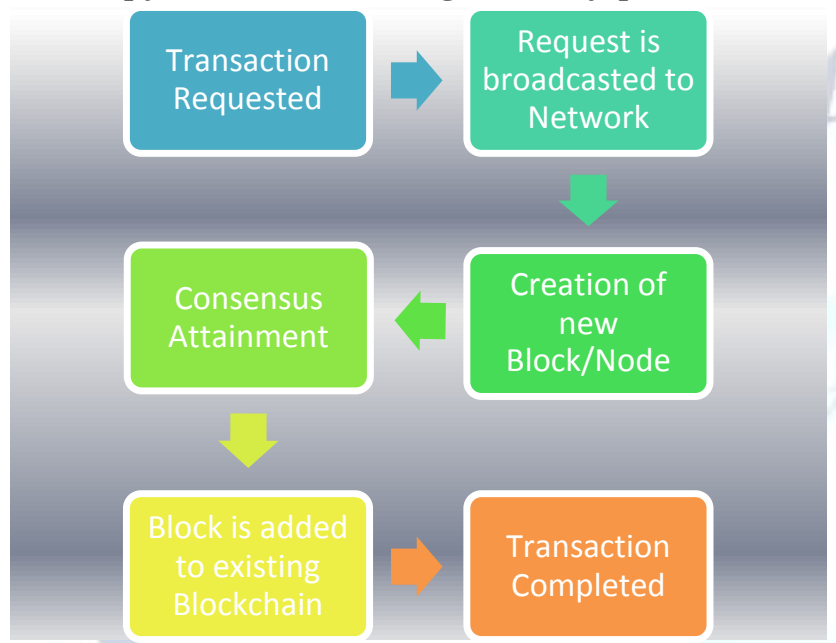

Figure1: Blockchain Transaction Process

There are three broader categories or types of blockchain exists which are namely Public, Private and Consortium Blockchain. Public or Permission-less blockchain provides accessibility of read and write to all the users of a network without acknowledging their credentials. Private or Permissioned blockchain grants read/write accessibility only after verifying the user identification. Consortium/Federated blockchain grants read feature to all the participants but only the authorized ones can make the changes.

A comparison between the various types of blockchain has been drawn. This comparison has been drawn after identifying some parameters in the below table.

Table- 1: Types of Blockchain

\section{TYPES OF BLOCKCHAIN}

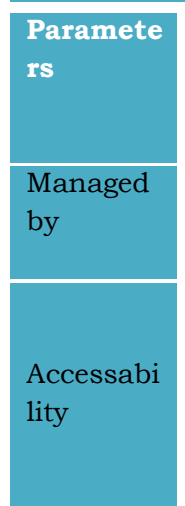

\begin{tabular}{l}
$\begin{array}{l}\text { Public } \\
\text { BlockChain/ } \\
\text { Permission-le } \\
\text { ss }\end{array}$ \\
No One \\
\hline $\begin{array}{l}\text { all participants } \\
\text { on the network } \\
\text { can perform } \\
\text { read and write } \\
\text { operations }\end{array}$ \\
\hline
\end{tabular}

\begin{tabular}{l|l|}
$\begin{array}{l}\text { Private } \\
\text { Blockchain/ } \\
\text { Permissione } \\
\text { d system }\end{array}$ & $\begin{array}{l}\text { Consortium/ } \\
\text { Federated } \\
\text { Blockchain }\end{array}$ \\
\hline $\begin{array}{l}\text { Single } \\
\text { Organization }\end{array}$ & $\begin{array}{l}\text { Group of } \\
\text { Organization } \\
\text { s }\end{array}$ \\
\hline $\begin{array}{l}\text { Authorized } \\
\text { participants } \\
\text { are All } \\
\text { pre-approved } \\
\text { for read/write } \\
\text { operations }\end{array}$ & $\begin{array}{l}\text { participants } \\
\text { can read the } \\
\text { data but only }\end{array}$ \\
a few
\end{tabular}

\begin{tabular}{|c|c|c|c|}
\hline & & & $\begin{array}{l}\text { ones can } \\
\text { write }\end{array}$ \\
\hline $\begin{array}{l}\text { Security } \\
\& \\
\text { consensu } \\
\text { s }\end{array}$ & $\begin{array}{l}\text { Complex due } \\
\text { to openness }\end{array}$ & $\begin{array}{l}\text { Relatively } \\
\text { Simple }\end{array}$ & Simple \\
\hline Usage & $\begin{array}{l}\text { Wide due to } \\
\text { their massive } \\
\text { decentralizatio } \\
\mathrm{n} \text { and } \\
\text { openness }\end{array}$ & $\begin{array}{l}\text { Limited to the } \\
\text { maintaining } \\
\text { organization }\end{array}$ & $\begin{array}{l}\text { Created for } \\
\text { group of } \\
\text { organizations } \\
\text { with similar } \\
\text { interest/need } \\
\text { s }\end{array}$ \\
\hline $\begin{array}{l}\text { Participa } \\
\text { nts } \\
\text { Status }\end{array}$ & Unidentified & Identified & Identified \\
\hline Security & Poor & High & High \\
\hline $\begin{array}{l}\text { Transacti } \\
\text { on Approv } \\
\text { al Time }\end{array}$ & $\begin{array}{l}\text { Relatively } \\
\text { longeer }\end{array}$ & Faster & Fastest \\
\hline
\end{tabular}

\section{Comparison OF VARIOUS AVAILABLE BLOCKCHAIN ARCHITECTURE}

Blockchain is gaining a lot of popularity in the research community these days. Researchers are coming up with their own architectures. A study of the various proposed architectures was carried out. A comparison of these proposed architectures has been made keeping in mind a few parameters. These parameters have been identified by studying the work given. The parameters for comparison are

Proposed By (who has proposed the architecture), Application areas (i.e. where it can be applied), Year of proposal, Title of Research Paper and where it has been published.

On studying the comparison it was found that the given below architecture are either suitable for any specific domain or are just provides a basic information of architecture process. Hence, a need for an architecture arises which can be applied over multiple domains or application areas. So we have proposed architecture for blockchain system which can be further applied over enormous domains. This has been discussed in the next section.

Table 2: Comparison of various Blockchain Architecture

\begin{tabular}{|c|c|c|c|c|c|}
\hline $\begin{array}{l}\text { PARAME } \\
\text { TERS }\end{array}$ & \multicolumn{2}{|c|}{$\begin{array}{l}\text { COMPARISION } \\
\text { ARCHITECTURES }\end{array}$} & VAF & US & LABLE \\
\hline $\begin{array}{l}\text { Proposed } \\
\text { By }\end{array}$ & $\begin{array}{l}\text { S. } \\
\text { Balakri } \\
\text { shnan, } \\
\text { J. Janet }\end{array}$ & $\begin{array}{l}\text { Ruizhe } \\
\text { Yang, F. } \\
\text { Richard } \\
\text { Yu, } \\
\text { Pengbo } \\
\text { Si, } \\
\text { Zhaoxin } \\
\text { Yang, } \\
\text { and } \\
\text { Yanhua } \\
\text { Zhang }\end{array}$ & $\begin{array}{l}\text { A. R. } \\
\text { Revathi, } \\
\text { Shwetth } \\
\text { a M }\end{array}$ & $\begin{array}{l}\text { Junyao } \\
\text { Wang, } \\
\text { Shenlin } \\
\text { g Wang, } \\
\text { Junqi } \\
\text { Guo, } \\
\text { Yancha } \\
\text { ng Du, } \\
\text { Shaochi } \\
\text { Cheng, } \\
\text { Xiangya }\end{array}$ & $\begin{array}{l}\text { Zibin } \\
\text { Zheng } \\
\text { Shaoa } \\
\text { n Xie, } \\
\text { Hongn } \\
\text { ing } \\
\text { Dai, } \\
\text { Xiang } \\
\text { ping } \\
\text { Chen, }\end{array}$ \\
\hline
\end{tabular}




\begin{tabular}{|c|c|c|c|c|c|}
\hline & & & & ng Li & $\begin{array}{l}\text { and } \\
\text { Huai } \\
\text { min } \\
\text { Wang }\end{array}$ \\
\hline $\begin{array}{l}\text { Applicati } \\
\text { on Area }\end{array}$ & $\begin{array}{l}\text { Crypto } \\
\text { curren } \\
\text { cy } \\
\text { (Digital } \\
\text { Curren } \\
\text { cy) }\end{array}$ & $\begin{array}{l}\text { Edge } \\
\text { Comput } \\
\text { ing } \\
\text { Systems }\end{array}$ & $\begin{array}{l}\text { Supply } \\
\text { Chain }\end{array}$ & $\begin{array}{l}\text { Intellect } \\
\text { ual } \\
\text { Propert } \\
\text { y }\end{array}$ & $\begin{array}{l}\text { Overvi } \\
\text { ew }\end{array}$ \\
\hline Year & 2019 & 2018 & 2019 & 2018 & 2017 \\
\hline $\begin{array}{l}\text { Title of } \\
\text { the Paper }\end{array}$ & $\begin{array}{l}\text { Blockc } \\
\text { hain } \\
\text { Techno } \\
\text { logy: } \\
\text { Basics, } \\
\text { Archite } \\
\text { cture, } \\
\text { use } \\
\text { cases } \\
\text { and } \\
\text { platfor } \\
\text { ms. }\end{array}$ & $\begin{array}{l}\text { Integrat } \\
\text { ed } \\
\text { Blockch } \\
\text { ain and } \\
\text { Edge } \\
\text { Comput } \\
\text { ing } \\
\text { Systems } \\
: \text { A } \\
\text { Survey, } \\
\text { Some } \\
\text { Researc } \\
\text { h Issues } \\
\text { and } \\
\text { Challen } \\
\text { ges }\end{array}$ & $\begin{array}{l}\text { Blockch } \\
\text { ain : An } \\
\text { Overvie } \\
\text { w }\end{array}$ & $\begin{array}{l}\text { A } \\
\text { Summa } \\
\text { ry of } \\
\text { Researc } \\
\mathrm{h} \text { on } \\
\text { Blockch } \\
\text { ain in } \\
\text { the } \\
\text { Field of } \\
\text { Intellect } \\
\text { ual } \\
\text { Propert } \\
y\end{array}$ & $\begin{array}{l}\text { An } \\
\text { Overvi } \\
\text { ew of } \\
\text { Block } \\
\text { chain } \\
\text { Techn } \\
\text { ology: } \\
\text { Archit } \\
\text { ecture } \\
\text { Conse } \\
\text { nsus, } \\
\text { and } \\
\text { Futur } \\
\text { e } \\
\text { Trend } \\
\text { s }\end{array}$ \\
\hline $\begin{array}{l}\text { Publishe } \\
\text { d At }\end{array}$ & $\begin{array}{l}\text { CSI } \\
\text { Comm } \\
\text { unicati } \\
\text { ons }\end{array}$ & $\begin{array}{l}\text { IEEE } \\
\text { Commu } \\
\text { nication } \\
\text { s } \\
\text { Surveys } \\
\& \\
\text { Tutorial } \\
\text { s }\end{array}$ & $\begin{array}{l}\text { CSI } \\
\text { Commu } \\
\text { nication } \\
\mathrm{s}\end{array}$ & $\begin{array}{l}\text { Inter } \\
\text { natio } \\
\text { nal } \\
\text { Confe } \\
\text { rence } \\
\text { on } \\
\text { Identi } \\
\text { ficatio } \\
\text { n, } \\
\text { Infor } \\
\text { matio } \\
\text { n and } \\
\text { Know } \\
\text { ledge } \\
\text { in the } \\
\text { Inter } \\
\text { net of } \\
\text { Thing } \\
\text { s, } \\
\text { Publi } \\
\text { shed } \\
\text { by } \\
\text { Elsevi } \\
\text { er }\end{array}$ & $\begin{array}{l}\text { IEEE } \\
6 \text { th } \\
\text { Intern } \\
\text { ationa } \\
1 \\
\text { Congr } \\
\text { ess on } \\
\text { Big } \\
\text { Data }\end{array}$ \\
\hline $\begin{array}{l}\text { Layered } \\
\text { Architect } \\
\text { ure }\end{array}$ & No & Yes & No & Yes & No \\
\hline
\end{tabular}

\subsection{Transaction Layer}

The Transaction layer holds the request of the user i.e. whenever a new transaction is requested by any user the blockchain process begins. The transaction requests will be gathered in this layer.

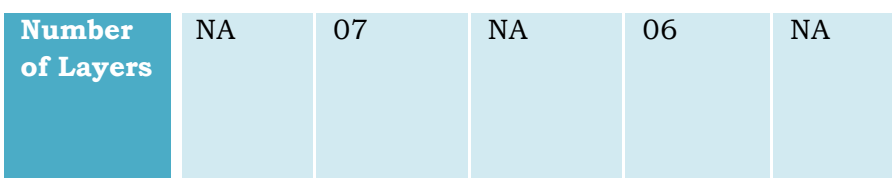

\section{Proposed 7 LAYer Blockchain ArChitecture}

Blockchain is a decentralized ledger over peer-to-peer (P2P) network. Every participant has its own identical replica of append-only ledger of digitally signed and encrypted transactions. Blockchain has gained its popularity with Bitcoin. [3] The Architecture for Blockchain has been proposed below. It comprises of 6 layers which have been discussed in detail below.

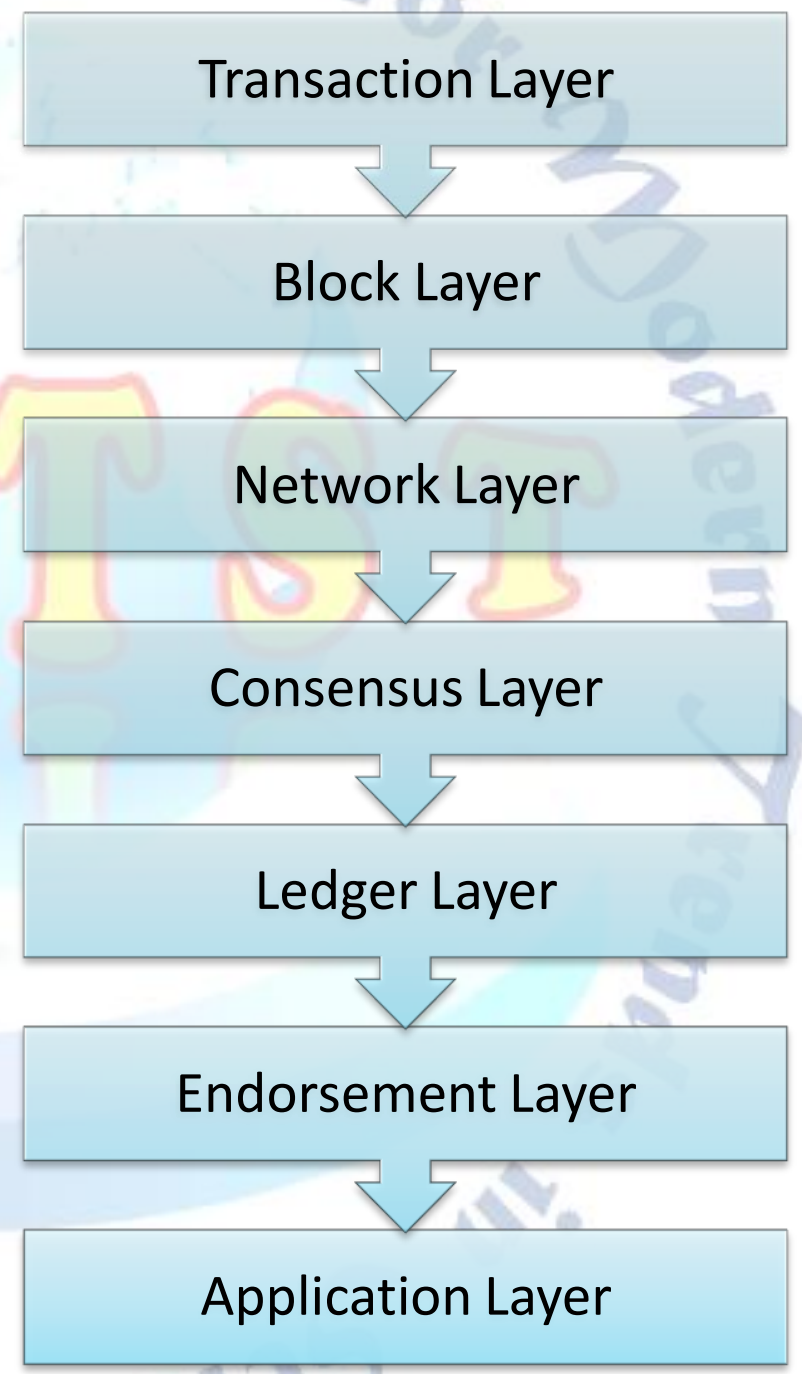

Figure 2: Blockchain Architecture

\subsection{Block Layer}

The block layer will be used for data block creation and storage. Data block consists of block header, index, timestamp, previous hash, hash and data. This layer encapsulates the data generated via transaction layer. Transactions are 
verified and packed into a block with a block header which further chained to the previous block.

\subsection{Network Layer}

The Network layer consists of peer-to-peer (P2P) network and communication mechanism. The main purpose of this layer is to broadcast data generated from lower layer. [iv] Transaction will be distributed to the neighbors by the use of networking mechanism, only the valid transactions are forwarded.

\subsection{Consensus Layer}

The Consensus Layer is also known as Protocol layer because this layer uses consensus algorithms to reach consensus between the unreliable nodes in environment. There are nine consensus mechanisms:

\section{- Proof-of-Work(PoW)}

PoW consensus protocol was proposed by Satoshi Nakamoto [1], according to which transactions are validated after participation of at least $51 \%$ nodes over original network [v] These validating nodes operate secretly and in huge number therefore these nodes have to mine the blocks for reading the transactions of the blockchain. After mining, new blocks are then permanently added to the blockchain. Hence, PoW protocols act as performance bottleneck in applications.

\section{- Proof-of-Stake(PoS)}

PoS consensus protocols resolve the high energy consumption of PoW. This protocol indentifies big stakeholders over the network and permits them to create new blocks. In this protocol validators are selected which reduces the security issues by prohibiting unknown validators therefore PoS is energy-efficient compared to PoW. [vi]

\section{- Byzantine Fault Tolerance (BFT)}

BFT uses a voting algorithm to remove validations from malicious nodes over the network because malicious nodes carry malicious intent code that can end up in the incorrect validations which can further corrupt the blockchain data. It is very challenging to find out malicious nodes and hence the implementation of BFT is very difficult. [vii]

\section{- Proof of Activity (PoAc)}

The PoAc protocol is an amalgamation of PoW and PoS. This protocol focus over rewarding the validators after solving mathematical problems which further results in increasing their stake in blockchain. The protocol then enables PoS algorithm for validators having suitable stake on the blockchain. PoAc has been established to be competent in terms of security, storage, and network communication. [viii]

\section{- Proof of Burn (PoB)}

The PoB protocol focuses on burning the coins or cryptocurrency over publically verified address. Users will be permitted to create new blocks after pay out with their coins and they be rewarded also in future. [ix] By the usage of PoB users can invest initially and can create theirs stocks in blockchain which can make them authorized validators. It in addition reduces the energy expenditure crisis of PoW. Moreover burning of coin reduces the total coin count on the blockchain which enhances the coin value progressively. In addition, coin burning balances the coin count on the blockchain network.

\section{- Proof of Elapsed Time (PoET)}

The PoET hunts a leader which has the power for new blocks creation rather than engaging all users in the process. Leader is chosen with the help of random leader selection algorithm in which a timer is added to every node and a leader is basically chosen with minimum timer expiry. [x] After creation of new blocks; digital signatures are transmitted over the network. Malicious users can be searched because if same nodes are elected as leaders repeatedly or if the minimum timer value is often assigned to same nodes. PoET may perhaps be helpful when used with delay-tolerant applications.

\section{- Proof of Capacity (PoC)}

The PoC protocol also known as proof of space protocol because it filter outs hard drive space on the nodes of the blockchain network. [xi] This protocol stores all potential nonce values on hard drive and to unlock the blocks it searches the matching nonce-hash pairs. The nodes with high disk space gains more stake.

\section{- Proof of Authority (PoA)}

PoA eliminates energy consumption issue of PoW and also eliminates the issue of dependency in PoS.

The basis behind PoA protocol is to give centralized reliable control to specific nodes that 
can together form the consensus based on majority votes to create new blocks on the network. [xii] Due to centralized control by valid stake holders PoA is pertinent for private networks, hence they have to undertake the legal aspects as well for granting the centralized control.

\section{- Proof of Importance (PoI)}

PoI protocols choose the validating nodes on the basis of frequency of maximum successful validations. Theme of both PoI and PoA is same only the criteria for choosing the validating node are different. [xiii] PoI protocol gains high trust amongst participating nodes because the significance of validators is recognized with their preceding successful validations.

\subsection{Ledger Layer}

Ledger layer consists of ledger created after authentication done by consensus layer. Ledger consists of chain of blocks.

\subsection{Endorsement Layer}

In this layer we can add crypto currency, smart contracts, records or any other related information to the ledger depending over the application.

\subsection{Application Layer}

This is the top most layers in blockchain system which includes ledgers, their respective cryptocurrency, smart contracts and other information also which would be further used in many applications like IOT, AI, education, tourism, smart cities, smart vehicles etc.

\section{APPLICATION AREAS OF BLOCKChaIN}

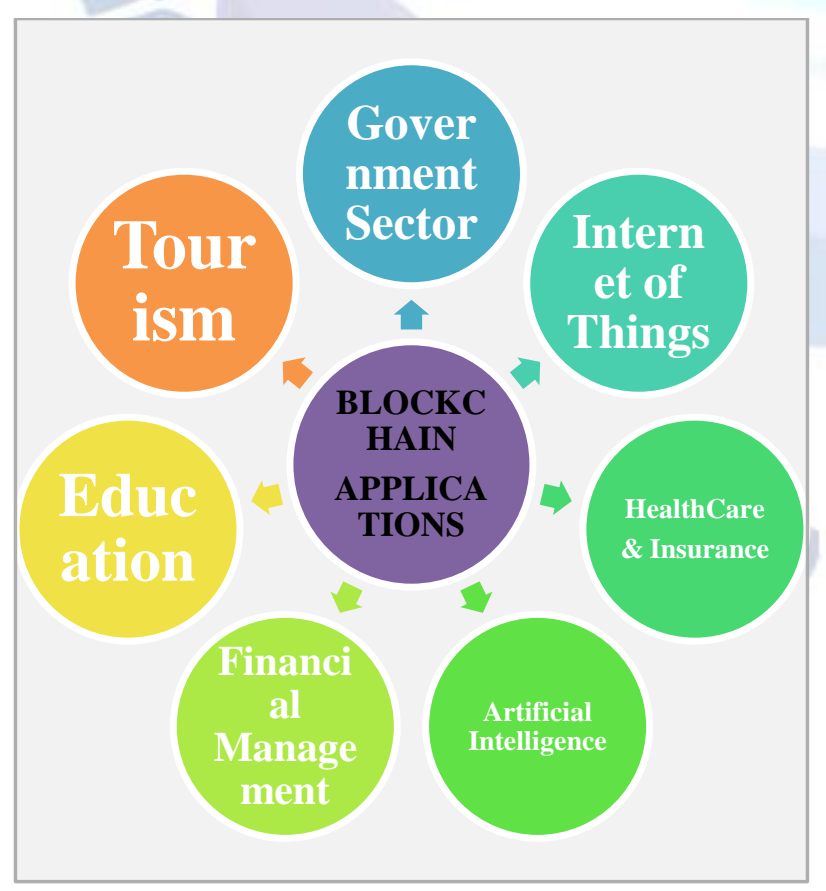

Figure 3: Blockchain Applications

\subsection{Government Sector/ Infrastructure \& Development}

Many government sectors are currently getting benefit from blockchain technology like Land registry system, International Trade Unit and Customs. In recent years, Swedish Lantmäteriet with the help of Telia Company has effectively tested a Blockchain Platform for Land Registry System. In this data units comprise of: (i) purchase/sale invoices and respective contracts, (ii) signatures of parties, (iii) identity documents of parties and (iv) owner information in Blockchain. [xiv] Government of Ghana also signed a MoU with IBM to develop a land registry system based on Blockchain. Recently, Andhra Pradesh has also started land registry system over blockchain.

\subsection{Internet of Things (IoT)}

Blockchain can transform many fields like healthcare, vehicles, energy consumption, cloud storage etc. $[\mathrm{xv}]$ "MarketsAndMarkets" a research firms predicts that the healthcare division of IoT will be worth $163.2 \mathrm{~B}$ by 2020 . [xvi]

From the past few years, researchers proposed the usage of blockchain technology to shield the privacy of health data. [xvii] By the usage of smart contracts over healthcare data a healthcare blockchain system can be created where sensors correspond with a smart device writes records over a confidential blockchain in consensus with Ethereum protocol. This sub-domain of IOT with blockchian is also referred as IoHT (Internet of Healthcare Things).

Similarly an IoV (Internet of Vehicles) network can also be formed with the help of blockchain technology which can play a key role in safe and convenient travel. [xviii] IoV can also enhance the lucidity and confidence in the used car segments which will further results into drop off frauds in this market. [xix]

Blockchain with IoT can also govern running of smart grid distribution network for electricity. [xx] Blockchain can facilitate traffic decisions over the smart grids so that power fluctuations can be minimized. [xxi].

\subsection{Health Care \& Insurance}

Medical data is conventionally saved by the medical institute only but sharing of data is vital requirement for drawing out crucial decisions which can further revolutionize the medical services radically. [xxii] Major barrier for sharing of medical data is accountability of privacy and security of records as this involves an individual's personal parameters. [ xxiii] The emergence of 
blockchain technology has resolved this issue to a very large amount as it is based on hash chain where a decentralized ledger is formed which is immutable and verifiable in nature.[ xxiv ] Blockchain technology helps many service providers, medical institutions and mainly patients for data access and sharing so that better alternatives can be sought.

\subsection{Artificial Intelligence (AI)}

The effective working of AI algorithm keenly relies over the quality and quantity of data or information available for making out inferences. These algorithms works better when a data repository is available for extraction, learning, inference-making and for taking final decision from data; though the repository source should be secure, trusted and reliable. [ $\mathrm{xxv}$ ] Blockchain resolves all these data repository issues of AI by providing a distributed shared ledger which contains cryptographically signed, validated and authentic data agreed on by all mining nodes. Moreover blockchain data can't be tampered by any external entity as one to make changes in blockchain network should be an authorized user of a blockchain P2P networks hence the integrity and resiliency is maintained. Smart contracts can do decision making and analytics part for machine learning algorithms, these results can be trusted and undisputed.[xxvi]By the usage of $\mathrm{AI}$ and Blockchain technology together we can create safe, immutable and decentralized system for highly sensitive AI-driven systems like AI-driven medical services, personal banking, financial assistance, trading, legal data handling and many more.

\subsection{Financial Management \& Accounting/ Trading/ Exchanges \& wallets/ Asset Management/ Payments \& Banking}

Blockchain technology is also known as future of financial and cyber security due to its crypto currencies. By the evolution of Bitcoin crypto currencies has changed the global market drastically. Bitcoin does not need any third party as it publicly shares the blockchain ledger. The involved users can use special software are called Bitcoin miners by which they can create a network to maintain the Blockchain. [xxvii] In Bitcoin mining process users can add new bitcoin currency and then transaction is broadcasted over the network.

\subsection{Education}

Blockchain technology is also transforming the education domain by its key features like: reliability, trust, security and efficiency. [xxviii] Nowadays, few universities and institutes have started with blockchain technology to support academic degree management and summative evaluation. Blockchain technology can prepare the entire script. In context of formal learning, it can include learning contents, their outcomes and achievements of students along with their academic certificates. Similarly, in the case of informal learning, the following parameters can be hold like their research experience, skills and interest of individual. A blockchain network can safely store all these parameters over their decentralized ledger from which various trends can be predicted for betterment of formal as well as informal evaluation for the education field.

\subsection{Tourism}

Tourism nowadays is majorly governed by Online Travel Services due to easy of ease and lucrative offers. But, these services are also suffering due to many problems like say double bookings, high foreign exchange rates, high commission charges and transaction fees for credit/debit cards usage. The most challenging thing is to maintain the security and privacy of any user as it involves very high financial exchange which is governed by payment service provider/third parties' travel agencies. Basically the travel agencies between customer and operators generate a high-profit margin for themselves which result is increased price for the user. Moreover, lot of user time is wasted in navigating over websites for searching out the best deals. Nowadays, many companies has started with blockchain technology to overcome these problems by which they provide a user Re-booking facility by a notification alert, if price drops; double booking problem is catered by smart contract system and blockchain transparency; user can even resell their tickets by the usage of $\mathrm{P} 2 \mathrm{P}$ network of blockchain and by searching out the cheapest deals by the collaboration of various major travel websites over a single network.

\section{FUTURE SCOPE \& CONCLUSION}

Blockchain has shown its potential for transforming conventional business with its key characteristics: decentralization, persistency, and anonymity and audit ability. In this paper, we present a comprehensive overview on blockchain technology and its process. The various types of blockchain have also been compared by identifying few distinguished parameters for them. Blockchain architecture has been proposed and a few architectures which have been proposed earlier have been compared. We proposed blockchain 
architecture also which can be applied further over various domains.

Nowadays, since blockchain based applications are springing up, these applications have been discussed and we plan to conduct in-depth investigations on blockchain-based applications in the future.

\section{REFERENCES}

[1] Bitcoin: A Peer-to-Peer Electronic Cash System by Nakamoto

[2] "Blockbench: A framework for analyzing private blockchains," by T.T. A. Dinh, J. Wang, G. Chen, R. Liu, B. C. Ooi, and K.-L. Tan, International Conference on Management of Data. ACM, 2017, pp. 1085-1100.

[3] "Integrated blockchain and edge computing systems: A survey, some research issues and challenges" by R.Yang

[4] "A summary of research on blockhchain in the field of intellectual property" by Wang, Guo.

[5] "Ethereum: A secure decentralised generalised transaction ledger," by G. Wood published in Ethereum project yellow paper, vol. 151, pp. 1-32, 2014.

[6] "Ppcoin: Peer-to-peer crypto- currency with proof of- stake," by S. King and S. Nadal, self-published paper, August, vol. $19,2012$.

[7] "Hyperledger fabric: a distributed operating system for permissioned blockchains," E. Androulaki, A. Barger, V. Bortnikov, C. Cachin, K. Christidis, A. De Caro, D. Enyeart, C. Ferris, G. Laventman, Y. Manevich et al., published at EuroSys Conference. ACM, 2018, p. 30.

[8] "proof of activity: Extending bitcoin's proof of work via proof of stake" by I. Bentov, C. Lee, A. Mizrahi, and M. Rosenfeld, ACM SIGMETRICS Performance Evaluation Review, vol. 42, no. 3, pp. 34-37, 2014.

[9] "Slimcoin | a cryprocurrency for long time," at SLM.pdf, vol. online, 2018.

[10] "On security analysis of proof-of-elapsed-time (poet)," L. Chen, L. Xu, N. Shah, Z. Gao, Y. Lu, and W. Shi, in SSS, 2017.

[11] F. Tschorsch and B. Scheuermann, "Bitcoin and beyond: A technical survey on decentralized digital currencies," IEEE Communications Surveys \& Tutorials, vol. 18, no. 3, pp. 2084-2123, 2016.

[12] S. D. Angelis, L. Aniello, R. Baldoni, F. Lombardi, A. Margheri, and V. Sassone, "Pbft vs proof-of-authority: Applying the cap theorem to permissioned blockchain," in ITASEC, 2018.

[13] "Nem $\hat{a}^{\top} \mathrm{A}$ 。S distributed ledger technology (blockchain)," URL https://nem.io/, vol. online, 2018.

[14] The Disruptive Blockchain: Types, Platforms and Applications Mahendra Kumar Shrivas 1, by Dr. Thomas Yeboah

[15] Blockchain and Its Role in the Internet of Things Mohamed Amine Ferrag, Leandros Maglaras, and Helge Janicke

[16] Griggs KN, Ossipova O, Kohlios CP, Baccarini AN, Howson EA, Hayajneh T (2018) Healthcare blockchain system using smart contracts for secure automated remote patient monitoring. J Med Syst 42:130

[17] Elmisery AM, Rho S, Botvich D (2016) A fog based middleware for automated compliance with OECD privacy principles in internet of healthcare things. IEEE Access 4:8418-8441
[18] Alam KM, Saini M, El Saddik A (2015) Toward social internet of vehicles: concept, architecture and applications. IEEE Access 3:343-357

[19] Brousmiche KL, Heno T, Poulain C, Dalmieres A, Hamida EB (2018) Digitizing, securing and sharing vehicles life-cycle over a consortium blockchain: lessons learned. In: 2018 9th IFIP international conference on new technologies, mobility and security (NTMS), pp 1-5. IEEE

[20] Ferrag MA (2017) an efficient privacy-preserving energy consumption scheme for smart grid communications. Telecommun Syst 66(4):671-688

[21] Knirsch F, Unterweger A, Eibl G, Engel D (2018) Privacy-preserving smart grid tariff decisions with blockchain-based smart contracts. In: Sustainable cloud and energy services. Springer, Cham, pp 85-116

[22] Blockchain-Based Medical Records Secure Storage and Medical Service Framework, Springer Science+Business Media, LLC, part of Springer Nature 2018

[23] Chen, T., and Zhong, S., Emergency access authorization for personally controlled online health care data. J. Med. Syst. 36(1):291,2012.

[24] Yue, X., Wang, H., Jin, D., Li, M., and Jiang, W., Healthcare Data Gateways: Found Healthcare Intelligence on Blockchain with Novel Privacy Risk Control. J. Med. Syst. 40(10):1-8, 2016.

[25] Blockchain for AI: Review and Open Research Challenges By K. SALAH1, M. H. REHMAN, N. NIZAMUDDIN1, and A. Al-Fuqaha.

[26] S. Janson, D. Merkle, and M. Middendorf, "A decentralization approach for swarm intelligence algorithms in networks applied to multi swarm pso," International Journal of intelligent computing and cybernetics, vol. 1 , no. 1 , pp. 25-45, 2008.

[27] Blockchain: Future of Financial and Cyber Security Sachchidanand Singh by IBM Software Lab Email: nirmala.online@gmail.com

[28] Exploring blockchain technology and its potential applications for education Guang Chen1,2, Bing Xu1, Manli Lu1 and Nian-Shing Chen3 